\title{
Metastatic Breast Lobular Carcinoma to Unusual Sites: A Report of Three Cases and Review of Literature
}

\author{
Hossameldin Abdallah ${ }^{\mathrm{a}}$, Amira Elwy ${ }^{\mathrm{b}, \mathrm{c}}$, Aya Alsayed ${ }^{\mathrm{d}}$, \\ Ahmed Rabea ${ }^{\text {a, }}$, Nesreen Magdy, e
}

\begin{abstract}
Invasive lobular carcinoma of the breast is the second most common type of invasive breast carcinoma. Invasive lobular carcinoma has an unusual pattern of metastases, which poses a diagnostic challenge for both clinicians and pathologists. We herein present three cases of breast invasive lobular carcinoma presented with metastasis to unusual sites, namely, uterus, colon and stomach. We recommend a higher index of suspicion in any case with breast cancer developing gastrointestinal tract or genital tract symptoms.
\end{abstract}

Keywords: Lobular carcinoma; GI metastases; Poorly cohesive carcinoma; Uterine bleeding; E- cadherin

\section{Introduction}

Breast invasive lobular carcinoma has a high frequency of distant metastases [1]. The most common sites of metastases are the bone, lung, pleura, soft tissue and liver [2]. Metastases to the female genital tract are rare $[2,3]$; and metastases to the gastrointestinal (GI) tract are even rarer $[4,5]$.

The incidence of extra-hepatic GI tract metastases of invasive lobular carcinoma ranges from $6 \%$ to $18 \%$, with the stomach being the most commonly affected site, followed by colon and rectum [3]. Clinically, metastatic invasive lobular carcinoma to the GI tract has a similar presentation to primary GI malignancies, and immunohistochemistry is essential to verify whether the tumor is primary or a metastatic [5].

Extra-genital primary malignancies metastasizing to the

Manuscript submitted June 30, 2020, accepted July 18, 2020

Published online August 6, 2020

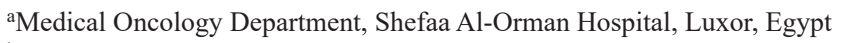
bPathology Department, South Egypt Cancer Institute, Assiut University, Assiut Governorate, Egypt

'Pathology Department, Shefaa Al-Orman Hospital, Luxor, Egypt

dMedical Oncology Department, National Cancer Institute, Cairo University, Egypt

${ }^{e}$ Corresponding Author: Nesreen Magdy, Pathology Department, National Cancer Institute, Cairo University, Egypt. Email: Nesreen.magdy@nci.cu.edu.eg

doi: https://doi.org/10.14740/jmc3538 female genital tract are uncommon, with most frequently affected site are the ovaries [3]. The most common source of metastatic involvement of the uterus is carcinoma of breast origin $(42.9 \%)$ [6].

We herein report two cases of metastatic lobular carcinoma to the GI tract (colon and stomach) and one case metastatic to the uterine corpus. We concluded that a thorough workup and a higher suspicion index of metastases should be adopted in cases with history of or current breast carcinoma, especially if it is of the invasive lobular carcinoma type, when developing GI tract or genital tract symptoms.

\section{Case Reports}

\section{Case 1}

A 59-year-old postmenopausal lady has presented to her primary care physician with progressive lower abdominal pain of 2 months duration, not associated with abnormal vaginal bleeding or discharge. Clinical examination revealed a bulky uterus and cervix. A transvaginal ultrasound showed an interstitial uterine fibroid of $16 \times 15 \times 10 \mathrm{~cm}$, with no extra uterine extension. Total abdominal hysterectomy and bilateral salpingo-oophorectomy (TAH-BSO) was performed.

The patient was referred to our hospital and histopathologic review of the TAH-BSO blocks revealed involvement of the endometrium, myometrium, fibroid and cervix by sheets of non-cohesive large epithelioid cells having eccentric nuclei and vacuolated cytoplasm (plasmacytoid/signet ring cells). The cells were positive by immunostaining for cytokeratin 7 (CK7), GATA3, estrogen receptor (ER) and progesterone receptor (PR), raising the possibility of breast origin. Other immunostains (desmin, cluster of differentiation 10 (CD10), actin and caldesmon) were negative (Fig. 1a-d).

The clinical examination revealed an upper outer quadrant (UOQ) right breast lesion measuring $5 \times 4 \mathrm{~cm}$, the Breast Imaging Reporting and Data System (BIRADS) 4 by bilateral sonomammography (BSM) with ipsilateral palpable lymph nodes. Left breast was unremarkable, clinically and radiologically. The biopsy of the right breast lesion was required, and it was diagnosed as invasive lobular carcinoma, with ER and PR positive and human epidermal growth factor receptor 2 (HER2) negative.

A metastatic workup was done (computed tomography 

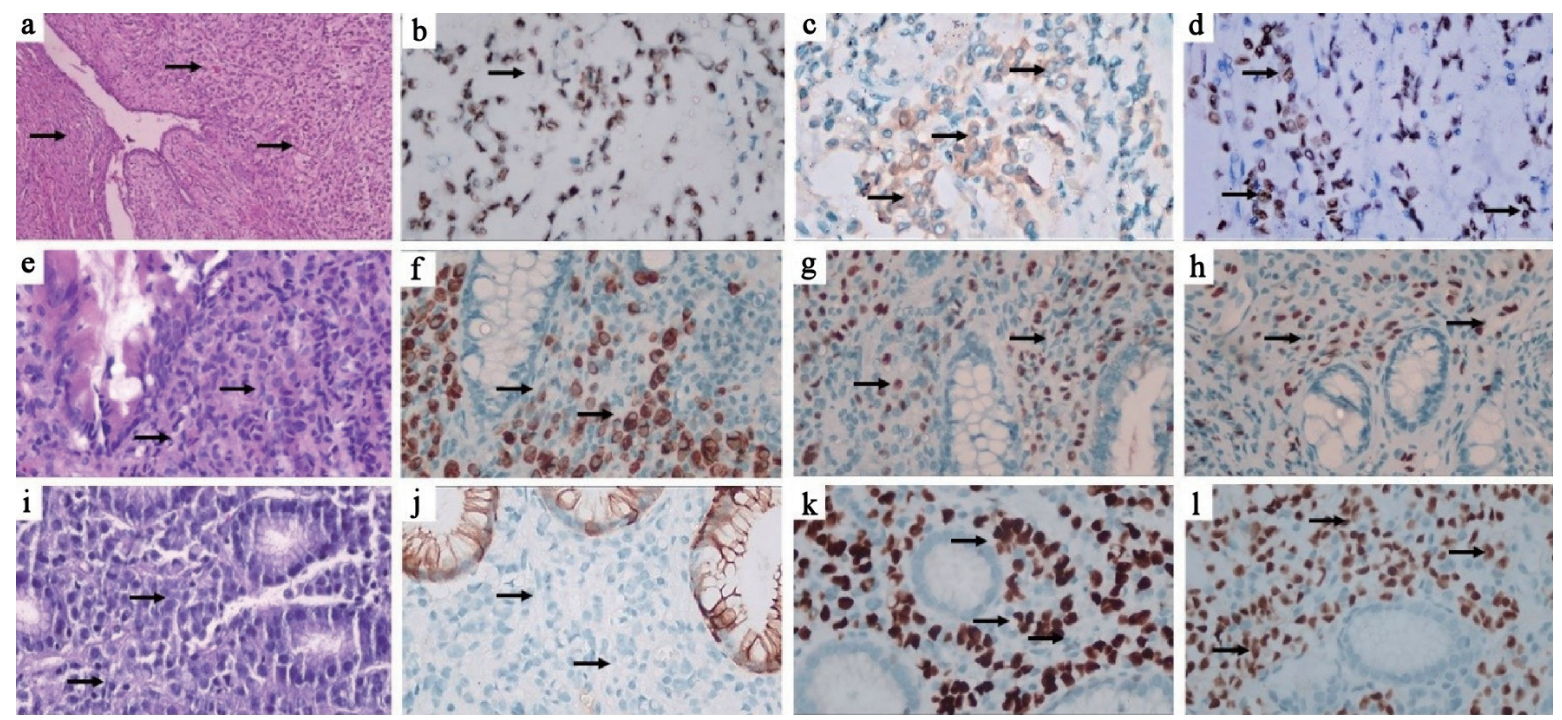

Figure 1. Case 1: (a) Hematoxylin and eosin (H\&E) stained section showing infiltration of the cervical wall by non-cohesive tumor cells (black arrows), $\times 100$. (b) Tumor cells show nuclear reaction for GATA3 (black arrow) × 400, (c) cytoplasmic reaction for mammaglobin (black arrows) $\times 400$ and (d) nuclear reaction for ER (black arrows) x 400. Case 2: (e) H\&E stained section of colonic mucosa diffusely infiltrated by tumor cells $(\times 100)$. (f) Tumor cells show cytoplasmic reaction for CK7 (black arrows), $\times$ 400 and (g) nuclear reaction for ER (black arrows), $\times 400$ and (h) GATA-3 (black arrows), $\times 400$. Case 3: (i) H\&E stained section showing infiltration of gastric mucosa by non-cohesive tumor cells (black arrows), $\times 400$. (j) Tumor cells are negative for CK20 (black arrows), which stains the surface mucosa, $\times 400$. (k) Tumor cells show positive nuclear reaction for ER (black arrows), $\times$ 400 and (I) GATA-3 (black arrows), × 400. CK: cytokeratin; ER: estrogen receptor.

(CT) and magnetic resonance imaging (MRI)), which revealed multiple enlarged para-aortic and iliac lymph nodes with cervical stump soft tissue mass. Bone scan revealed right scapular single metastatic osseous lesion. A decision of palliative chemotherapy was taken, due to her significant symptoms. Six cycles of FEC100 (5-fluorouracil $500 \mathrm{mg} / \mathrm{m}^{2}$ intravenous (IV), day 1 , epirubicin $100 \mathrm{mg} / \mathrm{m}^{2} \mathrm{IV}$, day 1 and cyclophosphamide $500 \mathrm{mg} / \mathrm{m}^{2}$ IV day 1 , every 21 days (q21d)) were given. Mid cycles evaluation revealed regressive disease of breast and pelvic masses. She received hormonal therapy post chemotherapy.

\section{Case 2}

A 66-year-old woman with a significant long history of right breast benign featuring mass has presented with remarkable weight loss over 4 months prior to accidental discovery of another right breast mass.

Physical examination revealed an upper inner quadrant (UIQ) breast with no tender mass. BSM showed two right breast masses, a benign featuring one $2.5 \times 3 \mathrm{~cm}$ (BIRADS 1 ) and a suspicious one $1 \times 1.5 \mathrm{~cm}$ (BIRADS 4), with bilateral suspicious axillary lymph nodes, the largest on the right side.

The suspicious right breast mass and a left axillary lymph node were biopsied, and revealing the invasive lobular carcinoma, positive for $\mathrm{CK}$. Immunostaining of ER and PR were strongly positive and HER-2 negative.

Bone scan revealed multiple osseous lesions. CT showed diffuse circumferential mural thickening of the ascending and transverse colon with a few reactionary regional lymph nodes. Endoscopic biopsy from the colonic lesion revealed metastatic invasive lobular carcinoma of breast origin, proved by positive immunostaining reaction for CK7, GATA-3 and ER, while negative for colon specific markers CK20 and CDX2 (Fig. 1e-h).

She completed six cycles of palliative chemotherapy, FEC100 (5-fluorouracil $500 \mathrm{mg} / \mathrm{m}^{2} \mathrm{IV}$, day 1, epirubicin 100 $\mathrm{mg} / \mathrm{m}^{2} \mathrm{IV}$, day 1 and cyclophosphamide $500 \mathrm{mg} / \mathrm{m}^{2} \mathrm{IV}$, day 1, q21d) with overall regressive disease. This was associated with improvement of her symptoms. She has been maintained on hormonal therapy with aromatase inhibitors and denosumab. She finished 18 months of treatment with stationary disease on regular follow-up.

\section{Case 3}

A 53-year-old woman presented with progressive increase of the abdominal girth and chronic dull aching pain. Examination revealed diffuse tenderness, abdominal distention and a palpable right breast UOQ mass of $1.5 \times 1.5 \mathrm{~cm}$ with no axillary lymph nodes.

MRI studies revealed multiple metastatic nodules all over the intestine, omentum, and peritoneal wall, bilateral ovarian enlargement, gastric wall thickening, ascites and extensive bone marrow abnormality.

Upper GI tract endoscopy revealed gastric wall thickening and a biopsy was taken. A guided biopsy was obtained from the abdominal lesions. Both were diagnosed as metastatic invasive lobular carcinoma of breast origin, immunostaining positive for CK7, GATA-3 and ER while negative for E-cadherin, CK20 and CDX2 (Fig. 1i-1).

She finished eight cycles of paclitaxel and carboplatin 
(paclitaxel $80 \mathrm{mg} / \mathrm{m}^{2} \mathrm{IV}$, day $1,8,15+$ carboplatin AUC 2 $\mathrm{IV}$, on day $1,8,15$; both $\mathrm{q} 28 \mathrm{~d}$ ). Interim CT studies evaluation revealed a stationary course, with significant improvement of her symptom. Then, she received hormonal treatment (anastrazole) after confirming her menopausal status. She will be evaluated every 3 months while on hormonal treatment.

\section{Discussion}

Female genital tract organs are at risk for the development of metastasis from remote primaries, yet this remains an uncommon condition. For that reason it poses a diagnostic challenge for both the clinicians and pathologists [7].

Metastatic emboli to the uterine corpus are unusual from extra genital sources. When metastases are detected, breast carcinoma is usually the primary source (42.9\%) [6]; and invasive lobular carcinoma is the most common type [8]. Metastasis to the cervix is even rarer attributed to its small size, the thick fibromuscular composition, and its limited blood supply [9].

Compared to invasive duct carcinoma, not otherwise specified (NOS), invasive lobular carcinoma has a distinctive metastatic patterns, including multiple metastasis sites, higher risk for liver metastases [10]. It has a higher tendency for meningeal, peritoneal, gastrointestinal or GI tract and uterine metastases [11].

One of the most consistent molecular alterations in invasive lobular carcinoma is the loss of the adhesion molecule Ecadherin. This explains the characteristic non-cohesive pattern of the tumor cells. Inactivation of this gene occurs as an early event in oncogenesis, as a result of somatic, truncating mutations, loss of heterozygosity $(\mathrm{LOH})$ and promoter methylation [1]. The lost E-cadherin facilitates the seeding of the tumor cells to remote sites $[12,13]$. This also explains the higher frequency of bilaterality in invasive lobular carcinoma cases [9].

It has been reported that development of metastasis in the GI or genital tracts occurs frequently after years of diagnosis of breast cancer while on hormonal treatment or during follow-up period [14-18]. However a few reported cases initially presented with distant metastasis with or without simultaneous primary invasive lobular carcinoma $[9,19]$. That highlights the rarity of the presentation of the aforementioned cases.

Abnormal vaginal bleeding has been repeatedly reported as the manifestation of metastatic invasive lobular carcinoma to the uterus $[2,9]$. In our first case, despite the huge size of the uterine mass and infiltration of the cervix, her presentation was insidious with only long history of lower abdominal pain without any reported bleeding. Similarly, in the second case, the colonic metastatic manifestations were subtle and discovered accidentally while performing metastatic workup. Nevertheless, in the literature, GI metastasis usually present with intestinal obstruction [20,21] or bleeding per rectum [19]. The presentation on the third case was rare as there was a gastric wall lesion with extensive abdominal wall and peritoneal lesions.

Invasive lobular carcinoma was associated with larger tumor size, older age, later stage, lower grade, ER/PR posi- tive and negative HER2, which were the characteristics of our three cases [10].

Although in Egypt there are some breast cancer early detection programs, yet neither of the patients had been enrolled in any, and unfortunately they presented later with distant metastases. This highlights the enormous importance of the breast cancer screening.

Initial presentation with metastatic breast cancer has a detrimental effect on the overall survival. However, there is no consensus regarding the survival prognosis of invasive lobular carcinoma when compared to invasive duct NOS. Studies reporting 5-year survival data described a superior outcome in invasive lobular carcinoma patients [22]. Whereas studies that conducted longer follow-up periods tend to show similar or even worse outcome compared to invasive duct carcinoma NOS [23].

Hormonal profile has a good prognostic impact on the survivorship of the breast cancer patients. It has been found that ER+/HER2- (including both Ki67 index high as well as low groups) and ER+/HER2 + tumors has a median survival time of 25 and 24 months, respectively from the onset of development of the metastasis [24].

Our recommendations is to have a higher index of suspicion in any case with a history of current breast cancer, especially invasive lobular carcinoma when developing GI tract or genital tract symptoms, even if mild or subtle, and to biopsy any developed GI tract or genital tract lesions with performing an extensive immunostaining panel including breast specific antibodies.

\section{Acknowledgments}

None to declare.

\section{Financial Disclosure}

None to declare.

\section{Conflict of Interest}

The authors declare no conflict of interest.

\section{Informed Consent}

Not applicable.

\section{Author Contributions}

Hossam Abdallah: collection and/or assembly of data, data analysis and interpretation, writing the article, critical revision of the article, and final approval of article. Amira Elwy: data analysis and interpretation, critical revision of the article, and final approval of article. Aya Alsayed: collection and/or assem- 
bly of data, critical revision of the article, and final approval of article. Ahmed Rabea: critical revision of the article, and final approval of article. Nesreen Magdy: research concept and design, collection and/or assembly of data, data analysis and interpretation, writing the article, critical revision of the article, and final approval of article.

\section{Data Availability}

The data supporting the findings of this study are available from the corresponding author upon reasonable request.

\section{References}

1. Lakhani MJ, Ellis SR, Schnitt IO, Tan SJ, van de Vijver PH. WHO classifications of tumors of the breast. 2012.

2. Berger AA, Matrai CE, Cigler T, Frey MK. Palliative hysterectomy for vaginal bleeding from breast cancer metastatic to the uterus. Ecancermedicalscience. 2018;12:811.

3. Arrangoiz R, Papavasiliou P, Dushkin H, Farma JM. Case report and literature review: Metastatic lobular carcinoma of the breast an unusual presentation. Int J Surg Case Rep. 2011;2(8):301-305.

4. Cherian N, Qureshi NA, Cairncross C, Solkar M. Invasive lobular breast carcinoma metastasising to the rectum. BMJ Case Rep. 2017;2017.

5. Abid A, Moffa C, Monga DK. Breast cancer metastasis to the GI tract may mimic primary gastric cancer. J Clin Oncol. 2013;31(7):e106-107.

6. Kumar NB, Hart WR. Metastases to the uterine corpus from extragenital cancers. A clinicopathologic study of 63 cases. Cancer. 1982;50(10):2163-2169.

7. Mazur MT, Hsueh S, Gersell DJ. Metastases to the female genital tract. Analysis of 325 cases. Cancer. 1984;53(9):1978-1984.

8. Abdalla AS, Lazarevska A, Omer MM, Tan E, Asaad A, Sathananthan S. Metastatic Breast cancer to the cervix presenting with abnormal vaginal bleeding during chemotherapy: a case report and literature review. Chirurgia (Bucur). 2018;113(4):564-570.

9. Lokadasan R, Ratheesan K, Sukumaran R, Nair SP. Metastatic lobular carcinoma of breast mimics primary cervix carcinoma: two case reports and a review of the literature. Ecancermedicalscience. 2015;9:571.

10. Chen Z, Yang J, Li S, Lv M, Shen Y, Wang B, Li P, et al. Invasive lobular carcinoma of the breast: A special histological type compared with invasive ductal carcinoma. PLoS One. 2017;12(9):e0182397.

11. Harris M, Howell A, Chrissohou M, Swindell RI, Hudson M, Sellwood RA. A comparison of the metastatic pattern of infiltrating lobular carcinoma and infiltrating duct carcinoma of the breast. Br J Cancer. 1984;50(1):23-30.

12. Lehr HA, Folpe A, Yaziji H, Kommoss F, Gown AM. Cytokeratin 8 immunostaining pattern and E-cadherin expression distinguish lobular from ductal breast carcinoma. Am J Clin Pathol. 2000;114(2):190-196.

13. Dabbs DJ, Schnitt SJ, Geyer FC, Weigelt B, Baehner FL, Decker T, Eusebi V, et al. Lobular neoplasia of the breast revisited with emphasis on the role of E-cadherin immunohistochemistry. Am J Surg Pathol. 2013;37(7):e1-11.

14. Geredeli C, Dogru O, Omeroglu E, Yilmaz F, Cicekci F. Gastric metastasis of triple negative invasive lobular carcinoma. Rare Tumors. 2015;7(2):5764.

15. Saranovic D, Kovac JD, Knezevic S, Susnjar S, Stefanovic AD, Saranovic DS, Artiko V, et al. Invasive lobular breast cancer presenting an unusual metastatic pattern in the form of peritoneal and rectal metastases: a case report. J Breast Cancer. 2011;14(3):247-250.

16. Jordan LA, Green L. Late breast cancer metastasis to the urinary bladder presenting with bilateral hydronephrosis. Radiol Case Rep. 2018;13(6):1238-1241.

17. Kesavan S, Lee IW. An unusual tumour metastasis to the cervix. Ann Acad Med Singapore. 2000;29(6):780-782.

18. Cift T, Aslan B, Bulut B, Ilvan S. Unusual uterine metastasis of invasive ductal carcinoma: A case report. Turk J Obstet Gynecol. 2016;13(3):164-166.

19. Shakoor MT, Ayub S, Mohindra R, Ayub Z, Ahad A. Unique presentations of invasive lobular breast cancer: a case series. Int J Biomed Sci. 2014;10(4):287-293.

20. Mistrangelo M, Cassoni P, Mistrangelo M, Castellano I, Codognotto E, Sapino A, Lamanna G, et al. Obstructive colon metastases from lobular breast cancer: report of a case and review of the literature. Tumori. 2011;97(6):800804.

21. Daniels IR, Layer GT, Chisholm EM. Bowel obstruction due to extrinsic compression by metastatic lobular carcinoma of the breast. J R Soc Promot Health. 2002;122(1):61-62.

22. Azim HA, Malek RA, Azim HA, Jr. Pathological features and prognosis of lobular carcinoma in Egyptian breast cancer patients. Womens Health (Lond). 2014;10(5):511518.

23. Pestalozzi BC, Zahrieh D, Mallon E, Gusterson BA, Price KN, Gelber RD, Holmberg SB, et al. Distinct clinical and prognostic features of infiltrating lobular carcinoma of the breast: combined results of 15 International Breast Cancer Study Group clinical trials. J Clin Oncol. 2008;26(18):3006-3014.

24. Savci-Heijink CD, Halfwerk H, Hooijer GK, Horlings HM, Wesseling J, van de Vijver MJ. Retrospective analysis of metastatic behaviour of breast cancer subtypes. Breast Cancer Res Treat. 2015;150(3):547-557. 